Military Technical College

Kobry Elkobbah,

Cairo, Egypt

May 25-27,2010

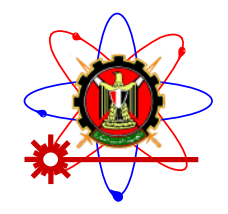

$5^{\text {th }}$ International Conference on Mathematics and Engineering Physics (ICMEP-5)

\title{
EM-20
}

\section{An Initial-Value Algorithm for Solving a Class of Non-Linear Singularly Perturbed Two-Point Boundary Value Problems}

\author{
E.R.El-zahar
}

\begin{abstract}
We consider non-linear singular perturbation problems of the form $\varepsilon y^{\prime \prime}(x)+p(y(x)) y^{\prime}(x)-q(x, y(x))=r(x), y(0)=\alpha, y(1)=\beta$ with a boundary layer at one end point. The method is distinguished by the following fact: The original problem is reduced to an asymptotically equivalent first order initial value problem (IVP). Then, an initial-value algorithm is applied to solve this IVP. The algorithm is based on the locally exact integration of a linearized problem on a non-uniform mesh. Two terms recurrence relation with controlled step size is obtained. Several problems are solved to demonstrate the applicability and efficiency of the algorithm. It is observed that the present method approximates the exact solution very well.

Keywords: Two-point boundary value problems; Singular perturbation problems; Boundary layer; Initial-value problems; Nonuniform mesh
\end{abstract}

\section{Introduction}

Singularly perturbed boundary-value problems (SPBVPs) are of common occurrence in many branches of applied mathematics such as fluid dynamics, elasticity, chemical reactor theory, etc. Solutions of such problems depend on a small positive parameter in such a way that the solution varies rapidly in some parts and varies slowly in some other parts. So, typically there are thin transition layers where the solutions can jump abruptly, while away from the layers the solution behaves regularly and vary slowly. So the numerical treatment of singular perturbation problems represents a major computational challenge. In general, the numerical solution of a boundary-value problem will be more difficult matter than the numerical solution of the corresponding initial-value problems. Hence, we prefer to convert the second-order problem into first-order problems. In fact, some numerical techniques employed for solving SPBVPs are based on the idea of replacing a two-point boundary value problem by two suitable initial-value problems. For example, Kadabajoo and Reddy [1] presented a novel initial-value technique for a class of nonlinear SPBVPs .In their method, the original problem is replaced by an asymptotically equivalent first-order initial-value problem which is then solved by the Runge-Kutta method. Gasparo and Macconi [2] considered a semilinear SPBVP which was integrated to obtain two first-order initial-value problems, and considered both the 
inner and outer solutions. The integration of these initial-value problems goes in opposite direction, and the first problem can be solved only if the solution of the second one is known. A similar matching idea combining the reduced problem and a WKB approximation for the full problem has also been employed by Gasparo and Macconi [3] for linear and semilinear SPBVPs. These matching ideas are based on the method of asymptotic expansions and on the work of Roberts [4] who considered the matching between inner and outer solutions at an unknown location which was determined iteratively. Robert's idea has been extended by Valanarasu and Ramanujam [5] for boundary value problems of singularly-perturbed systems of odes; these authors used exponentially-fitted methods for solving the singularly-perturbed initial value problem. Reddy and Chakravarthy [6] presented a method of reduction of order for solving linear and a class of nonlinear SPBVPs. In their method, the original problem is replaced by two first-order initial-value problems, which are then solved by the Runge-Kutta method in opposite directions. They later [7], introduced three initial-value problems which are independent of perturbation parameter for linear SPBVPs. In this paper, an initial-value method which is simple to use and easy to implement is presented for solving non-linear singular perturbation problems of the form $\varepsilon y^{\prime \prime}(x)+p(y(x)) y^{\prime}(x)-q(x, y(x))=r(x), \quad x \in[a, b]$, $y(a)=\alpha, \quad y(b)=\beta$ with a boundary layer at one end point. The original problem is reduced to an asymptotically equivalent first-order initial-value problem (IVP). Then, a variable step size initial-value algorithm is applied to solve this IVP in the inner region. The algorithm is derived based on the locally exact integration of a linearized problem over a non-uniform mesh. Two-term recurrence relation with controlled step size is obtained. Some numerical examples are given to illustrate the validity of the given method. It is observed that the present method approximates the exact solution if exists to great extent.

\section{Description of the method:}

Consider the non-linear singularly perturbed two-point boundary-value problem

$$
\varepsilon y^{\prime \prime}(x)+p(y(x)) y^{\prime}(x)-q(x, y(x))=r(x) ; \quad x \in[a, b],
$$

with the boundary conditions

$$
y(a)=\alpha \text { and } y(b)=\beta,
$$

where $\varepsilon$ is a small positive parameter $(0<\varepsilon \square 1), a, b, \alpha, \beta$ are given constants, $p(y(x))$, $q(x, y(x))$ and $r(x)$ are assumed to be sufficiently continuously differentiable functions. Furthermore, we assume that the problem (1) has a solution which displays a boundary layer in the neighborhood of $x=a$.

Equation (1.a) could be rewritten as

$$
\varepsilon y^{\prime \prime}(x)+f^{\prime}(y(x))=g(x, y(x)) ; \quad x \in[a, b],
$$

where

$$
f^{\prime}(y(x))=f_{y}(y(x)) y^{\prime}, f_{y}(y(x))=p(y(x)) \text { and } g(x, y(x))=r(x)+q(x, y(x)) .
$$

Now, let $u(x)$ be the solution of the reduced problem

$$
f^{\prime}(u(x))=g(x, u(x)) \text { with } u(b)=\beta \quad \text {. }
$$


Then an asymptotically approximation to the given Eq.(3) as follows:

$$
\varepsilon y^{\prime \prime}(x)+f^{\prime}(y(x))=g(x, u(x)) .
$$

Integrating Eq.(6) yeilds

$$
\varepsilon y^{\prime}(x)+f(y(x))=\int g(x, u(x)) d x
$$

Using Eq.(5), we get

$$
\varepsilon y^{\prime}(x)+f(y(x))=f(u(x))+K,
$$

where $k$ is an integration constant. In order to determine $k$, we introduce the condition that the reduced equation of (8) should satisfy the boundary condition at $x=b$. Thus, we get $K=0$. Hence, a first-order equation which is asymptotically equivalent to the second-order Eq. (1) was obtained.

$$
\varepsilon y^{\prime}(x)+f(y(x))=f(u(x)),
$$

with initial condition

$$
y(a)=\alpha .
$$

Remark1. If we obtain the analytical solutions of the IVPs (5) and (9), then we have an asymptotic analytical solution of the original singularly perturbed two-point boundary-value problem (1).

Equation(9) will be solved based on a locally exact integration of a linearized problem on a non-uniform mesh as follows.

Consider the interval $[a, b]$ and divide it into $N-1$ non-overlapping subintervals $I=\left[x_{i}, x_{i+1}\right]$, $i=1: N-1$, such that $x_{1}=a$ and $x_{N}=b$, where $N$ denotes the number of grid points. Eq. (9) may be approximated by

$$
\varepsilon y^{\prime}(x)=A+B\left(x-x_{i}\right)+C\left(y(x)-y\left(x_{i}\right)\right) ; \quad x \in I,
$$

where $\quad A=\left(f\left(u\left(x_{i}\right)\right)-f\left(y\left(x_{i}\right)\right)\right), B=\frac{d f}{d x}\left(u\left(x_{i}\right)\right), C=-\frac{d f}{d y}\left(y\left(x_{i}\right)\right)=-p\left(y\left(x_{i}\right)\right), \quad$ whose analytical solution may be readily obtained.

For example, if $C \neq 0$, and an initial condition $y\left(x_{i}\right)=\alpha_{i}$

$$
y(x)=\phi\left(x, x_{i}, \alpha_{i}\right), \quad x \in I,
$$

where

$$
\phi\left(x, x_{i}, \alpha_{i}\right)=\alpha_{i}-\frac{A}{C}-\frac{B}{C}\left(x-x_{i}\right)-\frac{\varepsilon B-(A C+\varepsilon B)}{C^{2}} e^{\frac{C\left(x-x_{i}\right)}{\varepsilon}} .
$$

Equation (11) represent the analytical solution of Eq.(10) and a piecewise analytical solution of Eq. (9). Starting with initial condition $y(a)=\alpha_{1}=\alpha$, we can obtain $y(x)$ for $x \in\left[a, x_{2}\right]$, and 
then obtain a new initial condition $\alpha_{2} \square y\left(x_{2}\right)$ to obtain the solution $y(x)$ for $x \in\left[x_{2}, x_{3}\right]$, and so on.

$$
y\left(x_{i+1}\right)=\phi\left(x_{i+1}, x_{i}, \alpha_{i}\right), \quad \alpha_{i}=y\left(x_{i}\right), \quad i=1: N-1 .
$$

The two-term recurrence relation, Eq.(12) is explicit, so that Eq.(9) can be easily solved directly by forward substitution.

Remark 2. If $C=0$, then $\phi\left(x, x_{i}, \alpha_{i}\right)=\alpha_{i}+\left(x-x_{i}\right)\left(2 A+B\left(x-x_{i}\right)\right) / 2 \varepsilon$.

\section{Mesh selection strategy}

A non-uniform grid is formed in such a way that one wants to get more information about the solution of the IVP (9) in the boundary layer region and obtain solution at fewer number of points in the outer region. This is quite natural because one would like to portray the behavior of the solution inside the boundary layer region. The required step size can be determined directly according to the variation of the solution within a X-step as follows [8,9]:

If we stand at a point $x_{i}$ and we want to determine a point $x_{i+1}$, which verifies $\left|y\left(x_{i+1}\right)-y\left(x_{i}\right)\right|=\delta$, where $\delta$ is a user's specified (constant) factor, then

$$
x_{i+1} \leq x_{i}+\left|\delta / y^{\prime}\left(x_{i}\right)\right|
$$

where

$$
y^{\prime}\left(x_{i}\right)=\phi^{\prime}\left(x_{i}, \alpha_{i}\right)
$$

Remark 3. The approximated first derivative in Eq. (14) can be obtained directly from Eq. (9), where

$$
y^{\prime}\left(x_{i}\right)=\left(f\left(u\left(x_{i}\right)\right)-f\left(y\left(x_{i}\right)\right)\right) / \varepsilon=A / \varepsilon .
$$

These details will be combined in the following algorithm:

\subsection{Algorithm steps}

Step I: Compute the solution of the reduced problem (5) by one of those

A) Analytical integration

B) Numerical integration method, where $u(x)$ can be considered as a linear piecewise continuous function.

Step II: Input $\varepsilon, \alpha, \delta$; Set index $=1, \mathrm{Y}(1)=\alpha_{1}=\alpha, x_{1}=a$.

Step III: While $x \leq 1$, compute

(i) $A=f\left(u\left(x_{1}\right)\right)-f\left(y\left(x_{1}\right)\right), \quad B=\frac{d f}{d x}\left(u\left(x_{1}\right)\right), \quad C=-\frac{d f}{d y}\left(y\left(x_{1}\right)\right)$;

(ii) $x_{2}=x_{1}+\delta /|A| / \varepsilon$

(iii) $y\left(x_{2}\right)=\phi\left(x_{2}, x_{1}, \alpha_{1}\right)$, from Eq. (11)

(iv) if $\left|y\left(x_{2}\right)-u\left(x_{2}\right)\right|<5 \varepsilon$, Stop

(v) $\mathrm{Y}($ index +1$)=y\left(x_{2}\right), \mathrm{X}($ index +1$)=x_{2}, x_{1}=x_{2} ; \alpha_{2}=y\left(x_{2}\right) ;$ index $=$ index +1 
Step IV: Plot the solution

(i) Plot (X, verses Y)

The algorithm is easily adaptable on computer; we present it in MATLAB environment as shown in Appendix A.

\section{Numerical examples}

To demonstrate the applicability of the method we applied it to three non-linear test problems. These problems are discussed in the literature and their approximate solutions are available for comparison.

Example 4.1. Consider the following non-linear example from O'Malley [10]

$$
\varepsilon y^{\prime \prime}(x)-y(x) y^{\prime}(x)=0 ; \quad x \in[-1,1],
$$

with $y(-1)=0$ and $y(1)=-1$. The problem (15) has a uniformly valid approximation [10] for comparison,

$$
y(x)=-[1-\exp (-(x+1) / \varepsilon] /[1+\exp (-(x+1) / \varepsilon] .
$$

The reduced problem solution is $u(x)=-1$. Hence; the corresponding initial-value problem is given by

$$
\varepsilon y^{\prime}(x)-(y(x))^{2} / 2=-1 / 2 \text { with } \quad y(-1)=0,
$$

which has a piecewise analytical solution given by

$$
y(x)= \begin{cases}-\left(x-x_{i}\right) / 2 \varepsilon, & \alpha_{i}=0, \\ \frac{1}{2 \alpha_{i}}\left(1+\alpha_{i}^{2}+\left(\alpha_{i}^{2}-1\right) e^{\alpha_{i}\left(x-x_{i}\right) / \varepsilon}\right), & \alpha_{i} \neq 0 .\end{cases}
$$

The piecewise solution error is shown in Fig. 1.

Example 4.2. Consider the following non-linear example from Kevorkian and Cole [11]

$$
\varepsilon y^{\prime \prime}(x)+y(x) y^{\prime}(x)-y(x)=0 ; \quad x \in[0,1],
$$

with $y(0)=-1$ and $y(1)=3.9995$. The problem (16) has a uniformly valid approximation [11] for comparison,

$$
y(x)=x+c_{1} \tanh \left(c_{1}\left(x / \varepsilon+c_{2}\right) / 2\right),
$$

where $c_{1}=2.9995$ and $c_{2}=\left(1 / c_{1}\right) \ln \left[\left(c_{1}-1\right) /\left(c_{1}+1\right)\right]$. 
The reduced problem solution is $u(x)=x+2.9995$, and the corresponding initial-value problem is given by

$$
\varepsilon y^{\prime}(x)+0.5(y(x))^{2}=0.5(x+2.9995)^{2} \text { with } \quad y(0)=-1 \text {, }
$$

which has a piecewise analytical solution given by

$$
y(x)= \begin{cases}u_{i}\left(x-x_{i}\right)(x+2.9995) / 2 \varepsilon, & \alpha_{i}=0, \\ \frac{1}{2 \alpha_{i}^{2}}\left(\alpha_{i}^{3}+u_{i}\left(\alpha_{i}\left(u_{i}+2\left(x-x_{i}-\varepsilon\right)\right)+\left(\alpha_{i}^{3}+2 \varepsilon u_{i}-\alpha_{i} u_{i}^{2}\right) e^{\alpha_{i}\left(x-x_{i}\right) / \varepsilon}\right),\right. & \alpha_{i} \neq 0 .\end{cases}
$$

The piecewise solution error is shown in Fig. 2.

Example 4.3. Consider the non-linear example from O'Malley [10] given by

$$
\varepsilon y^{\prime \prime}(x)+e^{y(x)} y^{\prime}(x)-\frac{\pi}{2} \sin (\pi x / 2) e^{2 y(x)}=0, \quad x \in[0,1],
$$

with $y(0)=0$ and $y(1)=0$. The problem has a uniformly valid approximation for comparison

$$
y(x)=-\ln \left[(1+\cos (\pi x / 2))\left(1-0.5 e^{-x / 2 \varepsilon}\right)\right] .
$$

The reduced problem solution is $u(x)=-\ln (1+\cos (\pi x / 2))$ and the corresponding initialvalue problem is given by

$$
\varepsilon y^{\prime}(x)+e^{y(x)}=\frac{1}{1+\cos (\pi x / 2)}, \quad y(0)=0 .
$$

which has a piecewise analytical solution given by

$$
y(x)=\alpha_{i}-1+e^{u_{i}-\alpha_{i}}\left[1+\frac{0.5 \pi \sin \left(0.5 \pi x_{i}\right)}{1+\cos \left(0.5 \pi x_{i}\right)}\left(x-x_{i}-\varepsilon e^{-\alpha_{i}}\left(1-e^{\alpha_{i}\left(x_{i}-x\right) / \varepsilon}\right)\right)-e^{\alpha_{i}\left(x_{i}-x\right) / \varepsilon}\right]+e^{\alpha_{i}\left(x_{i}-x\right) / \varepsilon} .
$$

By considering the given problems solutions as our exact solution, the maximum numerical solutions errors are given in tables. Tables 1 and 2 present the maximum error for the numerical solution obtained for each previous example over an approximated layer region $x \in\left[a, x_{p}\right]$,where $\left|y\left(x_{p}\right)-u\left(x_{p}\right)\right|>5 \varepsilon$, the required number of grid points $N_{\text {in }}$ over this region to achieve the specified factor $\delta$ at different values of $\varepsilon$, and the maximum error over the outer region $x \in\left[x_{p}, b\right]$ using the reduced problem.

The results indicate that the number of grid points required over the inner region is independent on the perturbation parameter $\varepsilon$, while it depends on the specified factor $\delta$ and can be approximated by 


$$
N_{i n} \square \frac{|y(0)-u(0)|}{\delta}+1
$$

Moreover, the numerical solutions errors over the inner region depend on both the perturbation parameter $\varepsilon$ and the specified factor $\delta$, while, at very small value of $\varepsilon,\left(\varepsilon<\delta^{2}\right)$, we have a second order of convergence as shown in fig. 4 .

Table 1. Maximum error and number of grid points $N_{i n}$ required over the inner region $\left[a, x_{p}\right]$ at $\delta=0.1$.

\begin{tabular}{|c|c|c|c|c|c|c|}
\hline$\varepsilon$ & & $10^{-3}$ & $10^{-5}$ & $10^{-7}$ & $10^{-9}$ & $10^{-11}$ \\
\hline \multirow{3}{*}{$\begin{array}{c}\text { Max error } \\
\text { (Example 4.1) }\end{array}$} & $x \in\left[-1, x_{p}\right]$ & $1.8454 \mathrm{e}-03$ & $1.8453 \mathrm{e}-03$ & $1.8453 \mathrm{e}-03$ & $1.8453 \mathrm{e}-03$ & $1.8453 \mathrm{e}-03$ \\
\hline & $x \in\left[x_{p}, 0\right]$ & $4.9110 \mathrm{e}-08$ & $3.3811 \mathrm{e}-10$ & $3.3802 \mathrm{e}-10$ & $3.3802 \mathrm{e}-10$ & $3.3802 \mathrm{e}-10$ \\
\hline & $N_{i n}$ & 11 & 12 & 12 & 12 & 12 \\
\hline \multirow{3}{*}{$\begin{array}{l}\text { Max error } \\
\text { (Example 4.2) }\end{array}$} & $x \in\left[0, x_{p}\right]$ & $6.9728 \mathrm{e}-004$ & $8.3172 \mathrm{e}-004$ & $8.3306 \mathrm{e}-004$ & 8.3308e-004 & 8.3308e-004 \\
\hline & $x \in\left[x_{p}, 1\right]$ & $4.4402 \mathrm{e}-16$ & $4.4400 \mathrm{e}-16$ & 0 & 0 & 0 \\
\hline & $N_{\text {in }}$ & 42 & 42 & 43 & 43 & 43 \\
\hline \multirow{3}{*}{$\begin{array}{l}\text { Max error } \\
\text { (Example 4.3) }\end{array}$} & $x \in\left[0, x_{p}\right]$ & $1.2000 \mathrm{e}-003$ & $1.2000 \mathrm{e}-003$ & $1.2000 \mathrm{e}-003$ & $1.2000 \mathrm{e}-003$ & $1.2000 \mathrm{e}-003$ \\
\hline & $x \in\left[x_{p}, 1\right]$ & $2.3635 \mathrm{e}-04$ & $1.6421 \mathrm{e}-05$ & 0 & 0 & 0 \\
\hline & $N_{\text {in }}$ & 8 & 9 & 10 & 10 & 10 \\
\hline
\end{tabular}

Table 2. Maximum error and number of grid points $N_{i n}$ required over the inner region $\left[a, x_{p}\right]$ at $\delta=0.01$.

\begin{tabular}{cllllll}
\hline$\varepsilon$ & & $10^{-3}$ & $10^{-5}$ & $10^{-7}$ & $10^{-9}$ & $10^{-11}$ \\
\hline \multirow{2}{*}{$\begin{array}{c}\text { Max error } \\
\text { (Example 4.1) }\end{array}$} & $x \in\left[0, x_{p}\right]$ & $\mathbf{1 . 9 7 6 4 e - 0 0 5}$ & $\mathbf{1 . 9 7 6 4 e - 0 0 5}$ & $\mathbf{1 . 9 7 6 4 e - 0 0 5}$ & $\mathbf{1 . 9 7 6 4 e - 0 0 5}$ & $\mathbf{1 . 9 7 6 4 e - 0 0 5}$ \\
& $x \in\left[x_{p}, 1\right]$ & $2.0000 \mathrm{e}-08$ & $1.8101 \mathrm{e}-10$ & $1.8101 \mathrm{e}-10$ & $1.8101 \mathrm{e}-10$ & 0 \\
& $N_{\text {in }}$ & 101 & 103 & 103 & 103 & 104 \\
\cline { 2 - 7 } & $x \in\left[-1, x_{p}\right]$ & $\mathbf{3 . 2 4 3 8 e - 0 0 4}$ & $\mathbf{7 . 0 5 3 0 e - 0 0 6}$ & $\mathbf{8 . 3 9 3 7 e - 0 0 6}$ & $\mathbf{8 . 4 0 7 2 e - 0 0 6}$ & $\mathbf{8 . 4 0 7 3 e - 0 0 6}$ \\
Max error & $x \in\left[x_{p}, 0\right]$ & $4.4414 \mathrm{e}-16$ & 0 & 0 & 0 & 0 \\
$\begin{array}{c}\text { (Example 4.2) } \\
\end{array}$ & $N_{\text {in }}$ & 402 & 403 & 404 & 404 & 404 \\
\cline { 2 - 7 } & $x \in\left[0, x_{p}\right]$ & $\mathbf{1 . 7 2 9 7 e - 0 0 5}$ & $\mathbf{1 . 5 0 4 5 e - 0 0 5}$ & $\mathbf{1 . 5 0 4 5 e - 0 0 5}$ & $\mathbf{1 . 5 0 4 5 e - 0 0 5}$ & $\mathbf{1 . 5 0 4 5 e - 0 0 5}$ \\
Max error & $x \in\left[x_{p}, 1\right]$ & $4.4011 \mathrm{e}-05$ & $9.7102 \mathrm{e}-06$ & $9.9700 \mathrm{e}-09$ & $9.9700 \mathrm{e}-09$ & $9.9700 \mathrm{e}-09$ \\
$($ Example 4.3) & $N_{\text {in }}$ & 71 & 72 & 72 & 73 & 73 \\
\hline
\end{tabular}




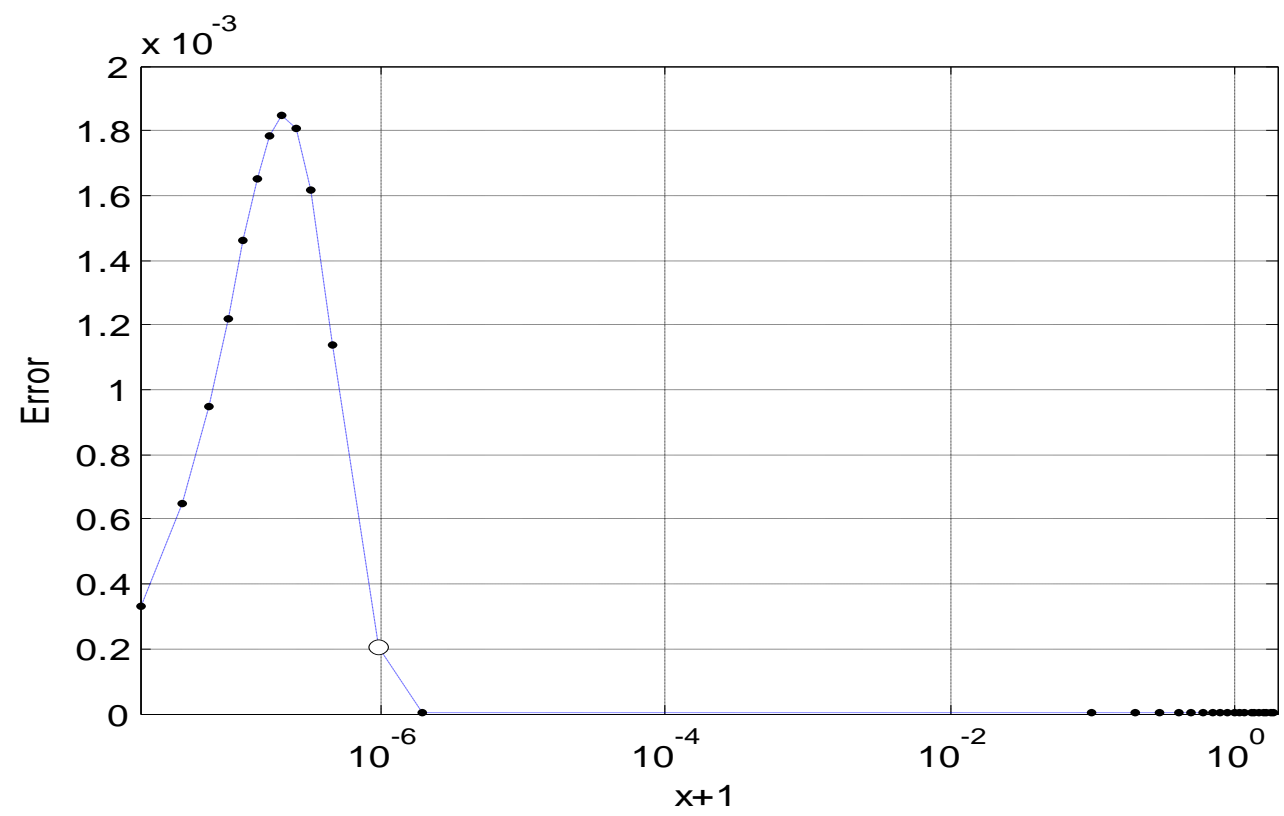

Fig. 1. The numerical solution error of Example 4.1 using the present algorithm at $\varepsilon=10^{-7}$.

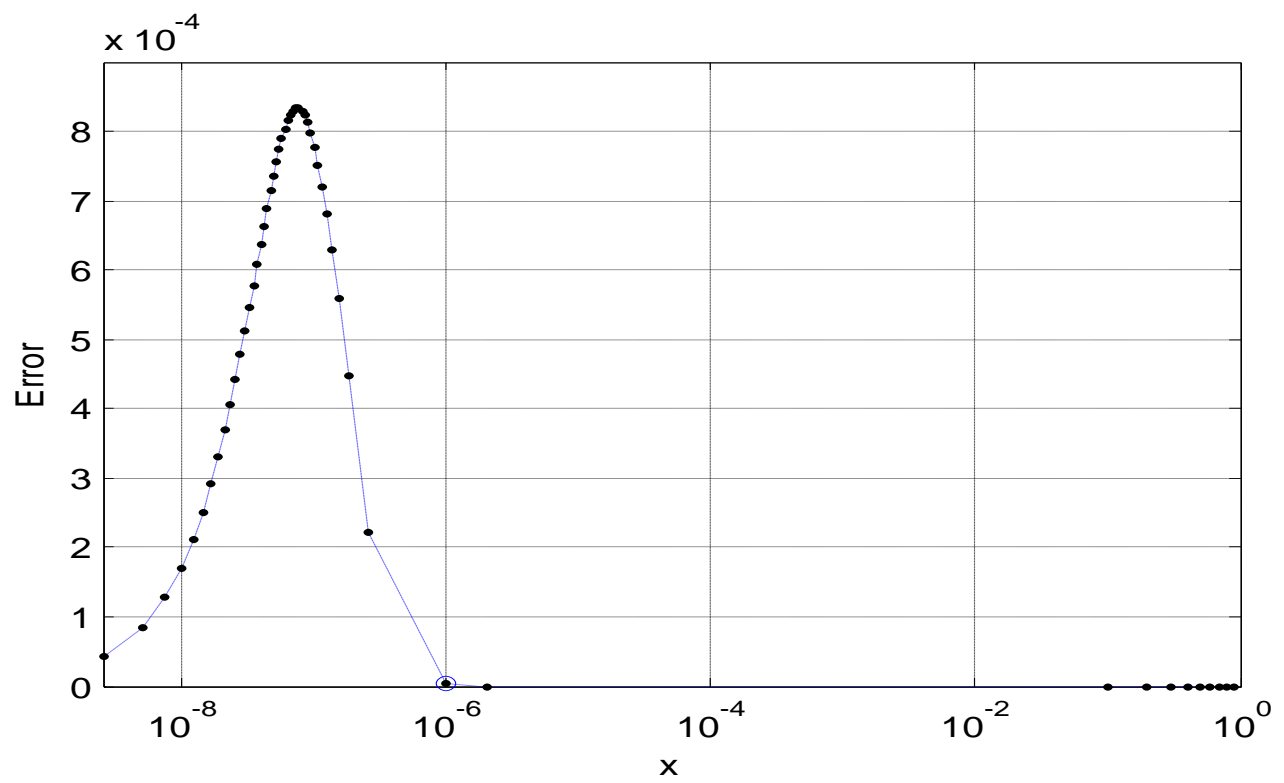

Fig. 2. The numerical solution error of Example 4.2 using the present algorithm at $\varepsilon=10^{-7}$. 


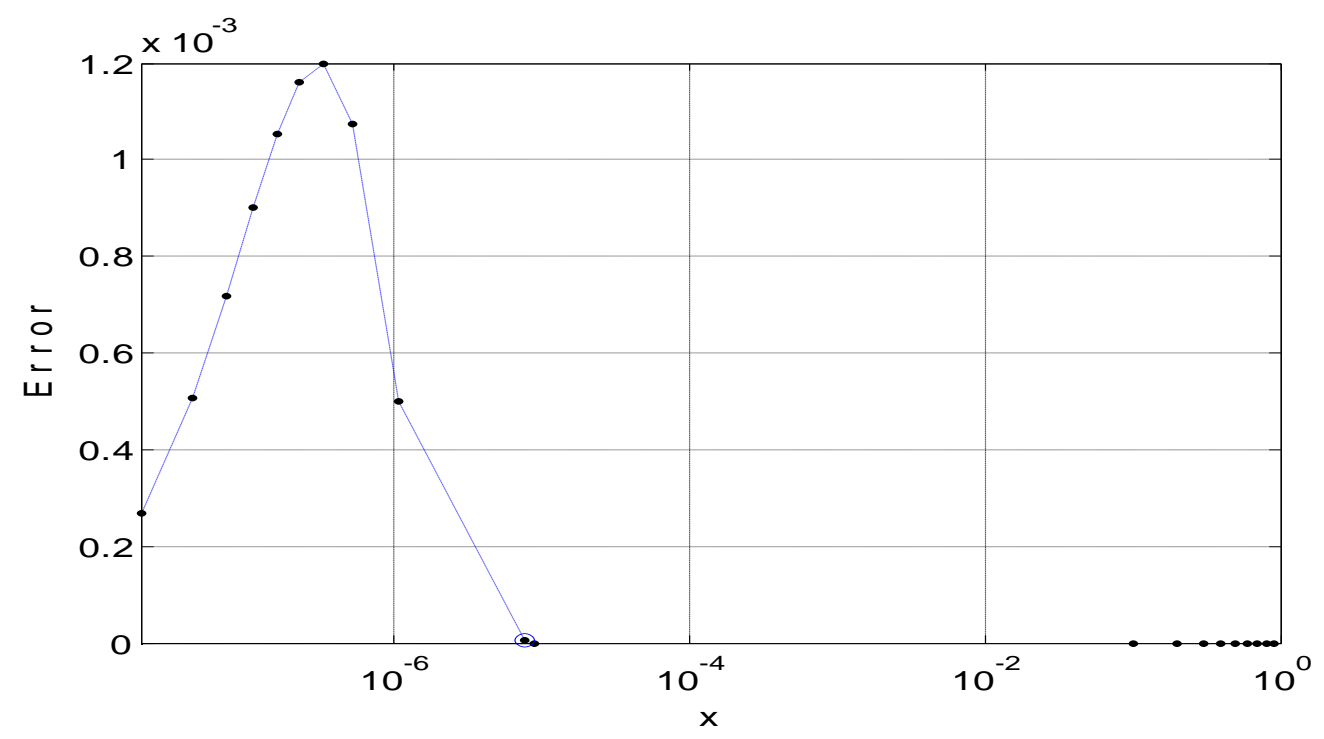

Fig.3. The numerical solution error of Example 4.3 using the present algorithm at $\varepsilon=10^{-7}$.



Fig.4. The computed order of convergence of the present method.

\section{Conclusions}

An initial-value algorithm with variable step size is presented for solving a class of non-linear singularly perturbed two-point boundary value problems with a boundary layer at one end point. The solution of the given problem is computed numerically by solving two initial-value problems. Two-term recurrence relation with controlled step size is obtained based on the locally exact integration of the linearized problem over a non-uniform mesh. The method is very easy to implement on any computer with minimum problem preparation. We have implemented it on three non-linear examples by taking different values of $\varepsilon$ and $\delta$. Piecewise approximations are presented for each example and numerical results are presented in tables and figures. The accuracy of the present method depends on both the perturbation parameter $\varepsilon$ and the specified factor $\delta$ and has a second order of convergence at very small values of $\varepsilon$. Moreover, the deduced equivalent initial- value problem enables us from obtaining an approximate value of the first derivative at the boundary layer point, which can be used in 
shooting techniques dealing with this type of problems. It can be observed that the present method approximates the exact solution very well.

\section{References}

[1] Kadabajoo, M.K. and Reddy,Y.N. "Initial value technique for a class of nonlinear singular perturbation problems" J. Optim. Theory Appl., vol.53, pp.395-406, (1987).

[2] Gasparo, M.G. and Maconi, M. "New initial value method for singularly perturbed boundary value problems" J. Optim. Theory Appl., vol.63, pp.213-224, (1989).

[3] Gasparo, M.G. and Maconi, M. "Initial value methods for second order singularly perturbed boundary value problems" J. Optim. Theory Appl., vol.66, pp.197-210, (1990).

[4] Roberts, S.M. "A boundary value technique for singular perturbation problems" J. Math. Anal. Appl., vol.87, pp.489-508, (1982).

[5] Valanarasu, T. and Ramanujam, N. "An asymptotic initial value method for boundary value problems for a system of singularly perturbed second order ordinary differential equations" Appl. Math. Comput., vol.147, pp.227-240, (2004).

[6] Reddy, Y.N. and Chakravarthy, P. P. "Method of reduction of order for solving singularly perturbed two-point boundary value problems" Appl. Math. Comput., vol.136, pp.27-45, (2003).

[7] Reddy, Y.N. and Chakravarthy, P. P. "An initial-value approach for solving singularly perturbed two-point boundary value problems" Appl. Math. Comput., vol. 155, pp. 95110, (2004).

[8] Ramos, J.I. "Linearization techniques for singularly-perturbed initial-value problems of ordinary differential equations" Appl. Math. Comput., vol. 163, pp.1143-1163, (2005).

[9] González-Pinto, S., Casasús, L. and González-Vera, P. "A numerical scheme to approximate the solution of a singularly perturbed nonlinear differential equation" $\mathrm{J}$. Comput. Appl. Math., vol.35, pp.217-225, (1991).

[10] O’Malley, R.E., Introduction to Singular Perturbations, Academic Press, N.Y., (1974).

[11] Kevorkian, J. and Cole, J.D., Perturbation Methods in Applied Mathematics, SpringerVerlag, N.Y., (1981).

\section{Appendix A}

clear all; close all;format long e

epsp $=1 \mathrm{e}-7 ;$ delta $=.1 ; \mathrm{hmax}=100 *$ epsp;tp $=100 *$ epsp;n=1;d=epsp $\mathrm{x} 0=-1$;

$\mathrm{x} 00=\mathrm{x} 0 ; \mathrm{x}=\mathrm{x} 0 ; ; \mathrm{y} 0=0 ; \mathrm{i}=0 ;$ while $\mathrm{x}<\mathrm{x} 00+\mathrm{tp}$

$\mathrm{i}=\mathrm{i}+1$; if $\mathrm{i}>500$; break; end

$\mathrm{y} 1=\left(-1 / 2+\mathrm{y} 0^{\wedge} 2 / 2\right) / \mathrm{epsp} ; \mathrm{h}=\mathrm{abs}(\operatorname{delta} /(\mathrm{y} 1+\mathrm{eps})) ; \mathrm{x}=\mathrm{x} 0+\mathrm{h} ; \mathrm{if} \mathrm{y} 0==0 ; \mathrm{y}=-(\mathrm{x}-\mathrm{x} 0) / 2 /$ epsp;end

if $\mathrm{y} 0 \sim=0 ; \mathrm{y}=1 / 2 / \mathrm{y} 0 *\left(\left(\mathrm{y} 0^{\wedge} 2+1\right)+\left(\mathrm{y} 0^{\wedge} 2-1\right)^{*} \exp (\mathrm{y} 0 *(\mathrm{x}-\mathrm{x} 0) / \mathrm{epsp})\right) ; \mathrm{u}=-1$; end

Yexact $=-(1-\exp (-(\mathrm{x}+1) / \mathrm{epsp})) /(1+\exp (-(\mathrm{x}+1) / \mathrm{epsp}))$

$\operatorname{er}(\mathrm{i})=\mathrm{abs}($ Yexact-y);xx(i)=x;yy(i)=y;u=-1;if abs(y-u)<5*epsp;break;end;

$\mathrm{x} 0=\mathrm{x} ; \mathrm{y} 0=\mathrm{y} ; \mathrm{end} ; \mathrm{xxx}=\mathrm{xx}($ end-n $)+\mathrm{d}: .1: 1 ; \mathrm{uuu}=0 * \mathrm{xxx}-1$;

yyy $=-(1-\exp (-(x x x+1) / e p s p)) /(1+\exp (-(x x x+1) / e p s p))$;

err=abs (yyy-uuu);semilogx $\left([\operatorname{xx}(1\right.$ :end-n $)+1, x x x+1],[\operatorname{er}(1:$ end-n $\left.), e r r],:^{\prime}\right)$

hold on; semilogx $(x x($ end-n)+1,er(end-n),'o');length(xx)

end 